\title{
Design and Dynamic Simulation in Scientific Computing Education
}

\author{
Lian Xue $\mathrm{e}^{1, \mathrm{a}^{*}}$ \\ ${ }^{1}$ School of Computer and Computing Science, Zhejiang University City College, Hangzhou, 310015, \\ P.R. China \\ $a^{*}$ xuel@zucc.edu.cn
}

Keywords: Scientific computing; Modeling; Design; Dynamic simulation

\begin{abstract}
This paper discusses teaching reform ideas in the "scientific computing" education by means of modeling and simulation, that can make students get a better understanding of the background, concept and design of each numerical algorithm in "scientific computing" education, and it will help them enhance their self-learning ability and exploration ability.
\end{abstract}

\section{Introduction}

In computer education, "scientific computing" is a way based on mathematical theory that uses computer-based tools for the purpose of calculating and studying on the computer to solve such as engineering problems, financial problems etc. It is closely related to computer development [1][2]. With the updating of the performance of computer hardware and software tools as well as the increasing popularity of computer applications, there is a need to make teaching content and teaching method in "scientific computing" education have an adjust and reform advancing with the times.

Modeling and simulation is a method which makes a practical problem into a mathematical model through mathematical theories and methods and then uses scientific computing analysis or computer means to solve the problem [3][4]. In recent years, with the depth of the quality education reform and the popularity of computer application, modeling and simulation becomes one searching direction of university computer education teaching reform.

In "scientific computing" education, we need to attach importance to modeling and simulation and make full use of modeling and simulation means according to the characteristic of teaching content in order to cultivate the ability of students to explore [5][6]. In this paper, a few suggestions are proposed in the teaching reform of "scientific computing" education applying modeling and simulation thought.

The rest of this paper is organized as follows. In the next section, modeling and simulation will be applied to help students understand the background of algorithm. Section 3 presents how to form basic concept of algorithm with modeling and simulation, followed with discussion about the use of GUI in computer in section 4. Finally, we conclude in Section 5.

\section{Using Modeling and Simulation to Help Students Understand the Background of Algorithm}

"Scientific computing" education nowadays is generally based on different actual problems and introduces a variety of categories of numerical methods. For a variety of numerical methods, we should be in accordance with the strict logic of the system and discuss them step-by-step in process of mathematical deduction, the geometric significance, the calculation formulas and convergence, the error analysis as well as application examples.

From students" point of view, "scientific computing" is about computing formula expressed by abstract mathematical symbols and proof of convergence theorem. And it's application is about calculating math problems in the mathematical analysis and advanced algebra or other math educations. In that way, the source and background of "scientific computing" can be easily concealed in the teaching process. But with the use of the modeling and simulation to deal with the actual problem 
programming, simulating, data analyzing in the computer, the technical background of numerical algorithm can be known, it will help enhance the enthusiasm of studying math and be able to design algorithm better.

For example: run a program in MATLAB (a computer software).

figure

set (gcf,'menubar','none')

axes ('position', [llllll)

$[x, y]=$ ginput

Then put your hand directly on the pop-up window, use the mouse clicks to select the required interpolation points, finally enter to get all the coordinates of interpolation points. But how can "appear" out of your hand on the screen as in Fig. 1 based on some coordinates? If you have studied the knowledge of the interpolation method, you can use the way of structuring "parameter curve" to analyze the problem with the acquaintance of the engineering background of interpolation. Parameter curve is a way that selects interpolation points in parameter intervals, and then uses cubic spline interpolation function approximating value at $\mathrm{m}$ points, and finally make the m-point graphic.

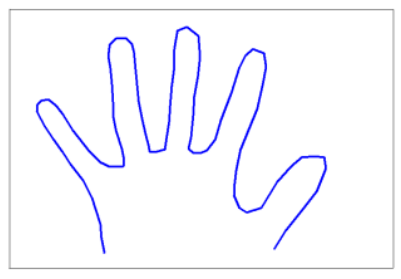

Figure 1. drawing up your hand on the screen based on some coordinates

In the case above which exploring the shape of hands you can find out more data by numerical methods through the analysis of small amounts of data to determine a variety of numerical methods adapted to the context of problem. This whole process is the application of modeling and simulation thought, and it also shows that modeling and simulation is a powerful vector to explore the ability of students to cultivate.

Modeling and simulation have the characteristics of intuitive, interoperability, repetitive, exploratory features in the occurrence and development of the process about math knowledge. In the process of solving of practical problems, using modeling and simulation means appropriately will make students have direct observation, hands-operation, consider in depth analysis, and repeatedly exploring. So that in the teaching of "scientific computing", we should pay full attention to modeling and simulation and then the ability of students to explore will be continuously upgraded.

\section{Using Modeling and Simulation to Help Students form Basic Concepts of Algorithms}

When solving math problems, Conventional way of thinking is a directional thinking from the conditions to the conclusions. But for some problems solving in that way, students still often cannot open the way of thinking or have the idea. Resorting to modeling and simulation means to explore the question can help us cross or bypass the obstacle of problem, overcome the difficulties of thinking and gradually form new ideas or new ways to solve the problem.

In many algorithms of "scientific computing" education, lots of concepts or knowledge are from engineering and economic fields. With the assistance of math experiment, we can guide the students to abstract the concept and process of numerical algorithm from the reality, explore and compare a variety of algorithms through the experiments, and then the conclusions will be summarized and organized into a meaningful whole. In turn, students will get an understanding of the algorithm and construct new concepts or new knowledge gradually. 
For example, the truncation error of "scientific computing" is an important resource to get numerical calculation error [7][8], but it is not the only one. It is a little hard for students to understand this one. However, if the experiment has been identified by way of $\mathrm{h}$ small enough, especially in the calculating of higher derivative, it will be found that when $\mathrm{h}$ after a small to a certain extent, the error of the numerical results will no longer reduce but be bigger (see Fig. 2)! Actually when step size $h$ is too small, the results of the error will be larger because of rounding errors. Taking advantage of these vivid visual experiments to start exploring enables students have a perceptual relationship on a more specific understanding to "truncation error" concept and its relationship with the round-off errors, and also enhances the students' ability to take the initiative to explore the question at the same time.

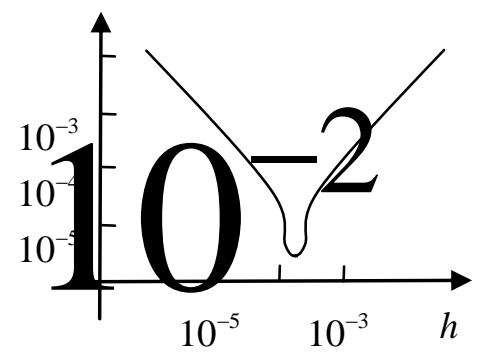

Figure 2. picture of relation of and error

It shows that in the process of teaching, turning to modeling and simulation means to guide students to use computer models, computer software, to go bold programming, testing and to explore is a way that makes the study of math become a proactive process to explore.

\section{Using Modeling and Simulation to Help Students Analyze Algorithm Deeply}

In the construction of "scientific computing" education, the software MATLAB is used in the process of modeling and simulation. MATLAB has powerful mathematical ability, convenient and practical functions of the graphics and the highly integration of language. It is becoming more and more widely in other areas of science and engineering and has a broader application prospects and potential for endless. Currently, MATLAB has become the most popular software tools for scientific and engineering computing.

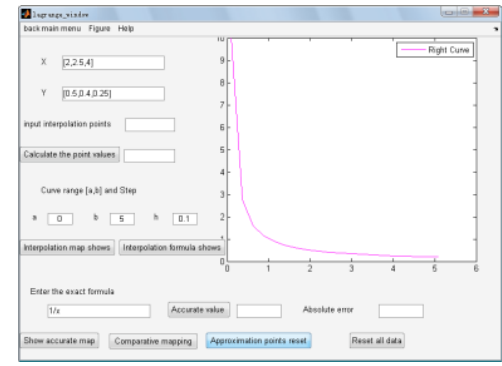

Figure 3. Lagrange Interpolation

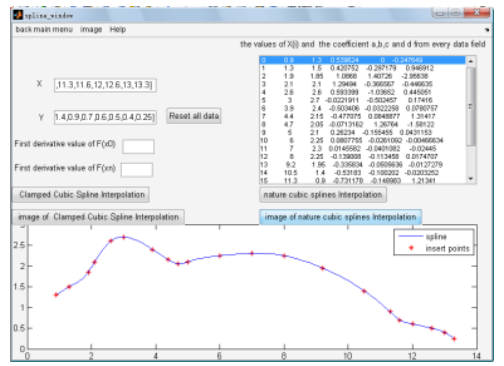

Figure 4. Spline Interpolation

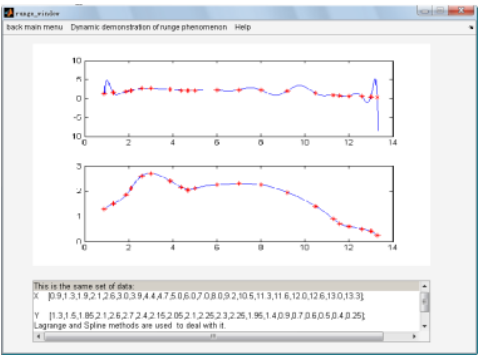

Figure 5. Runge Phenomenon

To comprehend the effect of algorithm with figures and results, we can turn to the GUI contact surface design in the GUIDE development system of MATLAB [9][10], which helps the users to realize the numerical methods of "scientific computing" well after learning it. GUI can show us required figures, codes, numerical values and error analysis by clicking controls in a form, so that users can understand each algorithm in the sight of computer implementation.

Take the interpolation as an example; we can explain advantage and disadvantage of 4 interpolation types (Lagrange Interpolation, Newton Interpolation, Piece Linear Interpolation, Cubic Interpolation). See Fig. 3 and Fig. 4, they shows respectively Lagrange Interpolation and Cubic Spline Interpolation. From the two figures, we can analyze easily their properties and error. In Fig. 5, the well-known 
"Runge Phenomenon" is displayed, which explain why Lagrange Interpolation is not stable and it will appear vibration in two end of interval. So that students will understand Lagrange Interpolation deeply.

\section{Conclusions}

Consequently, by modeling and simulation, we enable students discover the laws by themselves by incomplete summarizing with special cases; we let them propose conjecture and get the results at last. It is the way that contains preparation procedures, observation analysis, summarized guess. It's not only a meaningful "thought experiment", but also an effective way to forge the ability of exploration.

\section{References}

[1] Z.C.Shi, The Third Scientific Method- Scientific Computation in the Computing time, Tsinghua University Press, Beijing, 2000.

[2] Z.Li, Application of Math Experiment in the University Mathematics Teaching, Neijiang Technology, 28(2007)11-14.

[3] Q.Fu, A Brief Talk on the Students General Ability of Cultivating, Higher Engineering Education Research, 12(2002)86-88.

[4] D.Kincaid, Numerical Analysis :Mathematics of Scientific Computing, Tsinghua University Press, Beijing, 2003.

[5] J.Liu, Application of GUI in Matlab for the Information and Computing Science Specialty Teaching, Wuhan Technology, 20(2007)12-14.

[6] R.L.Burden, J.D.Faires, Numerical Analysis, Higher Education Press, BeiJing, 2001.

[7] C.N.Yang, On the application of math teaching and research, Yang Zhenning Speech Set, Nankai University Press, TianJin, 1985.

[8] Q.Fu, On the Students General cultivate the ability of students, Higher Engineering Education Research, 12(2002)86-88.

[9] Z.Li, Experimental Math at the University Mathematics Teaching, Neijiang Technology, 28(2007)11-12.

[10]Q.Fu, Brief discussion about cultivating the students' comprehensive ability, Researches in Higher Education of Engineering, 12(2002)86-88. 